\title{
Recurrence of Ventricular Fibrillation after Successful Conversion, May be Associated with Immediate Post-Shock Chest Compressions: A Case Report
}

\author{
Arthur Shiyovich $^{1^{*}}$, Alexander Gerovich $^{1}$ and Amos Katz ${ }^{1,2}$ \\ ${ }^{1}$ Department of Cardiology, Barzilai Medical Center, Ashkelon, Israel. \\ ${ }^{2}$ Faculty of Health Sciences, Ben-Gurion University of the Negev, Beer-Sheva, Israel.
}

Authors' contributions

This work was carried out in collaboration between all authors. Authors AG and AK designed the study. Authors AS and AG managed the literature searches. Author AS wrote the first draft of the manuscript. All authors read and approved the final manuscript.

\section{Case Study}

Received $15^{\text {th }}$ December 2012

Accepted $14^{\text {th }}$ February 2013

Published $28^{\text {th }}$ February 2013

\section{ABSTRACT}

Aims: Since 2005, cardiopulmonary resuscitation (CPR) guidelines advise immediately resuming CPR after a defibrillation shock to minimize CPR interruption. During resuscitation, the incidence of ventricular fibrillation (VF) recurrence is as high as $79 \%$. The aim of this report is to present a case of VF recurrence induced by chest compressions (CCs) following successful defibrillation of VF and to discuss the possible mechanisms that could be linked to this observation.

Case Presentation: A 57 year-old female suddenly collapsed and upon initiation of CPR, VF was observed. The patient was treated with 6 CPR-defibrillation cycles according to the current guidelines, after which she converted to normal sinus rhythm (NSR), but died following 9 in-hospital days. The monitor rhythms strips throughout resuscitation reveal restoration of NSR after the 4th defibrillation, yet CCs were resumed 1.3 seconds post DC shock and refibrillation closely followed. The first compression was timed exactly on the peak of the first post-shock sinus beat followed by refibrillation.

Discussion: possible mechanisms for the observed phenomenon include: creation of a long-short activation sequence by electric stimulation of the ventricles leading to VF recurrence, sudden stretch during a vulnerable window, which is determined by 
repolarization inhomogeneity and activation of mechano-sensitive ion channels, reperfusion arrhythmias (commonly ventricular tachycardia and PVCs) during restoration of coronary perfusion in acute myocardial infarction.

Conclusion: further evaluation of whether few second only of post shock pause and rhythm analysis might reduce the risk for such refibrillation and hence outweigh the minimal interruption of CCs is warranted.

Keywords: Cardiopulmonary resuscitation; Ventricular fibrillation; recurrence; chest compressions.

\section{INTRODUCTION}

Since 2005, guidelines advise immediately resuming cardiopulmonary resuscitation (CPR) for 2 consecutive minutes after a defibrillation shock to minimize CPR interruption. Such interruptions are an independent predictor of adverse outcomes, since they cause a drop in perfusion pressure and are associated with lower rates of return to spontaneous circulation (ROSC) [1]. Sell et al. [2] found that a preshock pause of less than 3 seconds is associated with a 6-fold increase in likelihood of ROSC, which when combined with a postshock pause of less than 6 seconds improved to a 13-fold increase in ROSC. Furthermore after VF is terminated most victims demonstrate a nonperfusing rhythm (pulseless electrical activity or asystole) for several minutes which is appropriately treated by CPR [3]. Hence, prolonged interruptions in chest compressions for analysis of a rhythm that is unlikely to require a shock do not seem to be justified [3].

During resuscitation, the incidence of ventricular fibrillation (VF) recurrence has been reported to be as high as $79 \%$ and significant inverse relation was found between the number of such refibrillations, and survival of out-of-hospital cardiac arrest [4]. Recent reports showed an association between VF recurrence and immediate resumption of chest compressions (CCs) following successful defibrillation [5,6]. We present a case of VF recurrence induced by CCs following successful defibrillation of VF and discuss possible mechanisms that could be linked to this observation.

\section{CASE REPORT}

A 57 year-old female collapsed following ambulatory dialysis treatment. Upon arrival, paramedics began CPR during which VF was observed. The patient was treated with 6 CPR and defibrillation cycles according to the current guidelines. Following the 6th shock the patient converted to normal sinus rhythm (NSR) and was brought unconscious to the emergency department and admitted at the intensive cardiac care unit. Therapeutic hypothermia was initiated and maintained for 24 hours. However the patient died after 9 inhospital days. Her past medical history revealed end stage renal disease treated by hemodialysis, hypertrophic cardiomyopathy, hypertention, hyperlipidemia and bipolar disorder.

An analysis of the monitor rhythms strips throughout resuscitation reveals that following the 4th defibrillation a sinus rhythm was restored, yet CCs were resumed 1.3 seconds post the DC shock without rhythm analysis (Fig. 1). Refibrillation closely followed initiation of CCs. The first compression was timed exactly on the peak of the first post-shock sinus beat followed by refibrillation. After the 6th DC Shock sinus rhythm was resumed after $1 / 3 \mathrm{sec}$ 
pause. This time CCs were not resumed and NSR with some pauses, peripheral pulse was palpated (110 bpm) and blood pressure was $113 / 69 \mathrm{~mm} / \mathrm{Hg}$.

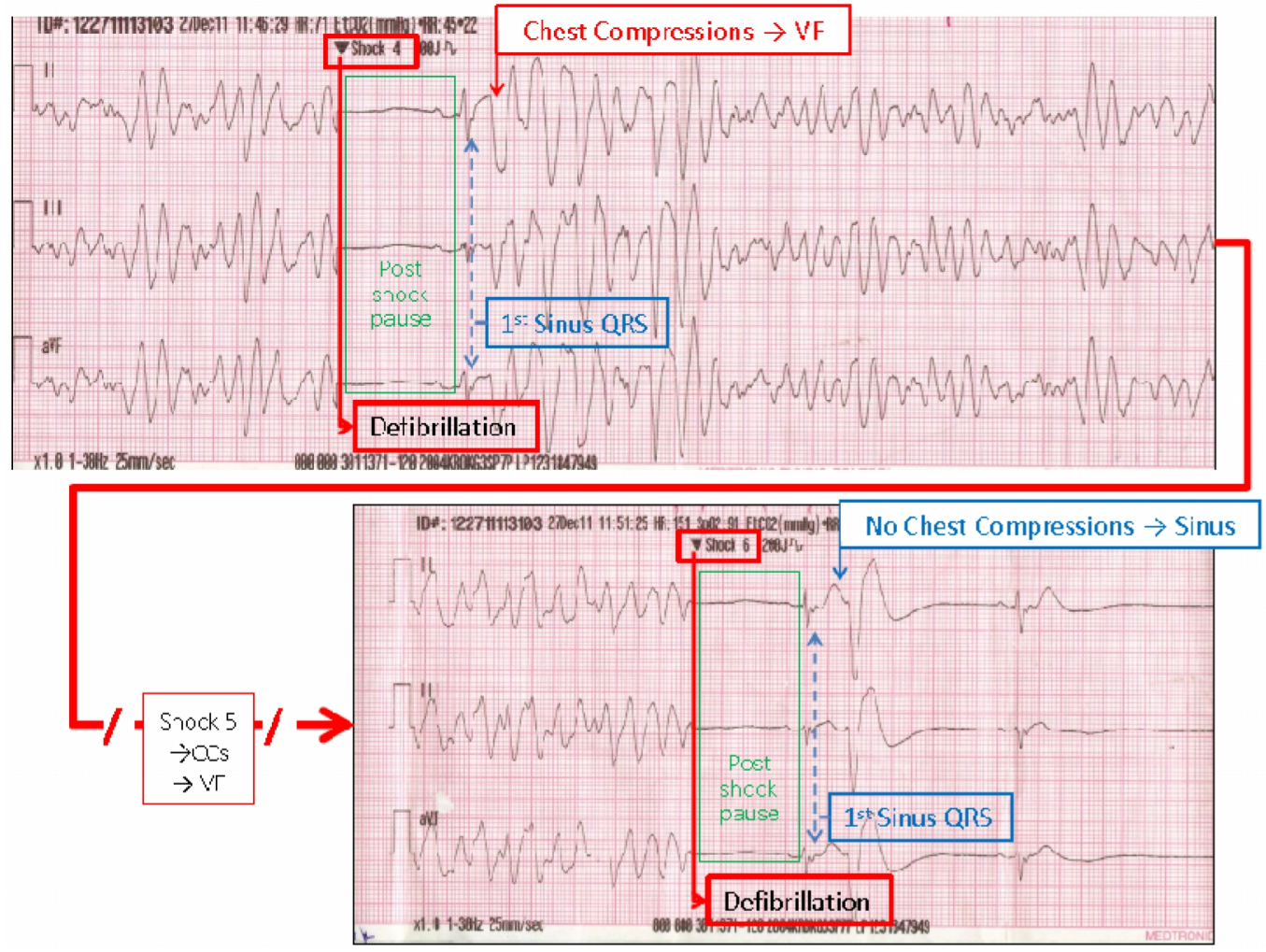

Fig. 1. The patient's monitor rhythm strip, during resuscitation

\section{Legend:}

A monitor rhythm strip during resuscitation displays VF treated by defibrillation (4th) resulting in restoration of NSR. Chest compressions were resumed 1.3 seconds post defibrillation without rhythm analysis and refibrillation closely followed. Successful defibrillation (6th and last) resulting in conversion to NSR with pauses for 5 seconds initially without resumption of CCs, followed by NSR of $110 \mathrm{bpm}$.

\section{DISCUSSION}

We report a case of recurrence of VF closely associated with immediate resumption of CCs following successful defibrillation of VF. Considering the patient's medical history it is highly plausible that the cardiac arrest was caused by an acute coronary event (e.g myocardial ischemia in a patient with significant coronary artery disease) Furthermore, an important potential precipitating etiology in this patient could be the dialysis treatment that causes intermittent fluctuations in the physiologic milieu with changes in blood pressure, electrolytes, and volume status and can adversely alter cardiovascular tone, function, and rhythms and ultimately lead to sudden cardiac death [7].

This case is consistent with the findings of Berdowski et al. [5] that the immediate resumption of CPR in accordance with resuscitation Guidelines 2005/2010, while significantly decreasing postshock pause and increasing the percentage of time CPR had 
been administered, also causes earlier VF recurrence and a trend toward longer-lasting VF. The authors found that the hazard ratio for VF recurrence in the first 2 seconds of CPR was 15.5 (95\% confidence interval, 5.63 to 57.7) compared with before CPR resumption [5]. After more than 8 seconds of CPR, the hazard of VF recurrence was similar to before CPR resumption 5. Consistently, in a recently reported animal study of long-duration VF $(>2.5$ $\mathrm{min}$ ), refibrillation occurred within the first $2 \mathrm{CCs}$ in 4 of 8 swine [6]. Capucci et al. studied 135 consecutive cases of out-of-hospital sudden cardiac death and found that CC was performed in 22 cases irrespective of the presence of spontaneous cardiac rhythm and in 14 cases CC was directly associated with VF induction [8]. Hess and White [9] retrospectively reviewed 67 patients with witnessed sudden cardiac arrest that were resuscitated by police/fire personnel. VF recurred during CCs in 16 of 32 patients, however, during post shock organized rhythm VF recurred spontaneously in $78 \%$ and during CCs in $22 \%$. Thus, contrary to the above reports the authors concluded that the recurrence of VF during a postshock organized rhythm is most commonly spontaneous and unrelated to CCs.

Osorio et al. showed that the electric stimulation of the ventricles by chest compressions created a long-short activation sequence leading to VF recurrence [6]. The mechanism by which a long-short sequence initiates VF is thought to be that the abrupt changes in the ventricular cycle length lead to increased dispersion of repolarization and the short cycle causes stimulation of the myocardium during the vulnerable period, creating unidirectional conduction block necessary for the initiation of re-entry that degenerates into VF [6]. Nonpenetrating blunt trauma to the chest wall (commotion cordis) could lead to sudden cardiac death due to the acute initiation of VF. VF may result from sudden stretch during a vulnerable window, which is determined by repolarization inhomogeneity. This results from the activation of mechano-sensitive ion channels. It seems highly possible that these are the mechanisms explaining VF induction by $\mathrm{CCs}$ in the current patient. Although it is theoretically possible that VF was induced by a premature ventricular contraction (PVC) during vulnerable period ("R on T") it seems less likely considering the characteristic morphology of CCs rather than PVC (Fig. 1).

A different mechanism could be reperfusion arrhythmias (commonly ventricular tachycardia and PVCs) during restoration of coronary perfusion in acute myocardial infarction [5], yet less likely here.

Limitation: consistently with the conclusions of Hess and White [9] it is possible that this observation of VF recurrence might happen irrespective of the commencement of CCs.

\subsection{Clinical Implications}

Although current CPR guidelines clearly decrease perishock pause and increase CPR time during resuscitation it may also increase VF recurrence. It is possible that few second only of post shock pause and rhythm analysis might significantly reduce the risk for such refibrillation and hence outweigh the minimal interruption of CCs. Additional studies to evaluate the effect of these factors on patient outcomes and to explore alternative approaches (e.g. rhythm identification during CCs) are warranted, and could improve future guidelines survival.

\section{CONSENT}

In this paper, only published data from the literature were used for description. Thus, a statement of patient consent is not applicable for this paper. 


\section{ETHICAL APPROVAL}

Since data from the literature, only, were used for description, ethical approval is not applicable to this paper. This study is not against the public interest. All authors hereby declare that all description have been performed in accordance with the ethical standards laid down in the 1964 Declaration of Helsinki.

\section{COMPETING INTERESTS}

Authors have declared no competing interests.

\section{REFERENCES}

1. Cunningham LM, Mattu A, O'Connor RE, Brady WJ. Cardiopulmonary resuscitation for cardiac arrest: the importance of uninterrupted chest compressions in cardiac arrest resuscitation. Am J Emerg Med. 2012;30(8):1630-8.

2. Sell RE, Sarno R, Lawrence B, Castillo EM, Fisher R, et al. Minimizing pre- and postdefibrillation pauses increases the likelihood of return of spontaneous circulation (ROSC). Resuscitation. 2010;81(7):822-5.

3. Hazinski MF, Nadkarni VM, Hickey RW, O'Connor R, Becker LB, Zaritsky A. Major changes in the 2005 AHA Guidelines for CPR and ECC: reaching the tipping point for change. Circulation. 2005;112(24 Suppl):IV206-11.

4. van Alem AP, Post J, Koster RW. VF recurrence: characteristics and patient outcome in out-of-hospital cardiac arrest. Resuscitation. 2003;59(2):181-8.

5. Berdowski J, Tijssen JG, Koster RW. Chest compressions cause recurrence of ventricular fibrillation after the first successful conversion by defibrillation in out-ofhospital cardiac arrest. Circ Arrhythm Electrophysiol. 2010;3(1):72-8.

6. Osorio J, Dosdall DJ, Robichaux RP Jr., Tabereaux PB, Ideker RE. In a swine model, chest compressions cause ventricular capture and, by means of a long-short sequence, ventricular fibrillation. Circ Arrhythm Electrophysiol. 2008;1(4):282-9.

7. Alpert MA. Sudden cardiac arrest and sudden cardiac death on dialysis: Epidemiology, evaluation, treatment, and prevention. Hemodial Int. 2011;15 Suppl 1:S22-9.

8. Capucci A AD, Bennati S, Piepoli M, Vilani G, Arvedi M, Bardy G. Ventricular fibrillation triggered by thoracic compression during out-of-hospital cardiac arrest resuscitation in the Piacenza Vita Project. J Am Coll Cardiol. 2004;43:302A.

9. Hess EP, White RD. Ventricular fibrillation is not provoked by chest compression during post-shock organized rhythms in out-of-hospital cardiac arrest. Resuscitation. 2005;66(1):7-11.

(c) 2013 Shiyovich et al.; This is an Open Access article distributed under the terms of the Creative Commons Attribution License (http://creativecommons.org/licenses/by/3.0), which permits unrestricted use, distribution, and reproduction in any medium, provided the original work is properly cited.

Peer-review history:

The peer review history for this paper can be accessed here: http://www.sciencedomain.org/review-history.php?iid=194\&id=12\&aid=1012 